From the Duke University Medical Center, Durham, NC; and the University of Texas M. D. Anderson Cancer Center, Houston, TX

Submitted February 18, 2010; accepted July 21, 2010; published online ahead of print at www.jco.org on October 4, 2010

Supported by National Institutes of Health Grants No. R01-CA97222-05 (J.H.S.), 5 P50 NS20023 and R37 CA 011898 (D.D.B.), and 5P50 CA108786 (D.D.B., J.H.S.); by grants from the American Brain Tumor Association, Accelerate Brain Cancer Cure, and the Brain Tumor Society (J.H.S.); and Commonwealth Cancer Foundation, the Adam Sliger Foundation, Dr. Marnie Rose Foundation, Anthony Bullock III Foundation, and Golfers Against Cancer (A.B.H.).

Both J.H.S. and A.B.H. contributed equally to this work.

Authors' disclosures of potential conflicts of interest and author contributions are found at the end of this article.

Clinical Trials repository link available on JCO.org.

Corresponding author: Darell D. Bigner $\mathrm{MD}, \mathrm{PhD}$, Preston Robert Tisch Brain

Tumor Center at Duke, Duke University Medical Center, DUMC 3156, 177 MSRB1, Durham, NC 27710; e-mail: bigne001@mc.duke.edu.

(C) 2010 by American Society of Clinical Oncology

0732-183X/10/2831-4722/\$20.00

DOI: 10.1200/JCO.2010.28.6963

\title{
Immunologic Escape After Prolonged Progression-Free Survival With Epidermal Growth Factor Receptor Variant III Peptide Vaccination in Patients With Newly Diagnosed Glioblastoma
}

John H. Sampson, Amy B. Heimberger, Gary E. Archer, Kenneth D. Aldape, Allan H. Friedman, Henry S. Friedman, Mark R. Gilbert, James E. Herndon II, Roger E. McLendon, Duane A. Mitchell, David A. Reardon, Raymond Sawaya, Robert J. Schmittling, Weiming Shi, James J. Vredenburgh, and Darell D. Bigner

See accompanying editorial on page 4670

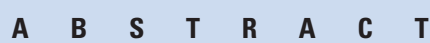

\section{Purpose}

Immunologic targeting of tumor-specific gene mutations may allow precise eradication of neoplastic cells without toxicity. Epidermal growth factor receptor variant III (EGFRvIII) is a constitutively activated and immunogenic mutation not expressed in normal tissues but widely expressed in glioblastoma multiforme (GBM) and other neoplasms.

\section{Patients and Methods}

A phase II, multicenter trial was undertaken to assess the immunogenicity of an EGFRvIII-targeted peptide vaccine and to estimate the progression-free survival (PFS) and overall survival (OS) of vaccinated patients with newly diagnosed EGFRvII-expressing GBM with minimal residual disease. Intradermal vaccinations were given until toxicity or tumor progression was observed. Sample size was calculated to differentiate between PFS rates of $20 \%$ and $40 \% 6$ months after vaccination.

\section{Results}

There were no symptomatic autoimmune reactions. The 6-month PFS rate after vaccination was $67 \%(95 \% \mathrm{Cl}, 40 \%$ to $83 \%)$ and after diagnosis was $94 \%(95 \% \mathrm{Cl}, 67 \%$ to $99 \% ; n=18)$. The median OS was 26.0 months $(95 \% \mathrm{Cl}, 21.0$ to 47.7 months). After adjustment for age and Karnofsky performance status, the OS of vaccinated patients was greater than that observed in a control group matched for eligibility criteria, prognostic factors, and temozolomide treatment (hazard ratio, 5.3; $P=.0013 ; \mathrm{n}=17)$. The development of specific antibody $(P=.025)$ or delayed-type hypersensitivity $(P=.03)$ responses to EGFRv/ll had a significant effect on OS. At recurrence, $82 \%(95 \% \mathrm{Cl}, 48 \%$ to $97 \%)$ of patients had lost EGFRvIll expression $(P<.001)$.

\section{Conclusion}

EGFRv III-targeted vaccination in patients with GBM warrants investigation in a phase III, randomized trial.

\section{J Clin Oncol 28:4722-4729. (C) 2010 by American Society of Clinical Oncology}

\section{INTRODUCTION}

Glioblastoma multiforme (GBM), the most common primary malignant neoplasm of the CNS, remains universally fatal. Patients with newly diagnosed GBM have a median overall survival (OS) of only 14.6 months despite maximal surgical resection, conformal radiation, ${ }^{1}$ and systemic chemotherapy. ${ }^{2}$ These conventional modalities lack specificity and, as a result, are limited by damage to normal tissue. ${ }^{1}$ Immunologic recognition of tumor-specific mutations, however, holds the promise of more precisely eliminating neoplastic cells.
The mutated epidermal growth factor receptor variant III (EGFRvIII) is a cell surface protein containing a tumor-specific epitope reportedly expressed on approximately one third of $\mathrm{GBMs}^{3,4}$ and a broad array of neoplasms in other tissues, including the breast, lung, and head and neck. ${ }^{5-8}$ It is not found in any normal tissues. ${ }^{9}$ EGFRvIII is characterized by a consistent in-frame deletion of 801 base pairs from the extracellular domain that splits a codon and produces a novel glycine at the fusion junction and approximates two normally distant parts of the protein. This mutation encodes a protein with a constitutively active tyrosine kinase $^{10}$ that 
enhances tumorigenicity ${ }^{11,12}$ and tumor cell migration ${ }^{13}$ and confers radiation and chemotherapeutic resistance to tumor cells. ${ }^{14-16}$ For patients with GBM who survive 1 year or longer after diagnosis, the expression of EGFR $v I I$ is also an independent negative prognostic indicator of survival. ${ }^{4}$ Thus, several factors make EGFRvIII an ideal target for antitumor immunotherapy.

On the basis of preclinical data supporting the safety and potential efficacy of an EGFRvIII-targeted vaccine, ${ }^{17,18}$ a phase II, multicenter, prospective trial was undertaken to assess the immunogenicity of an EGFR $v I I$-targeted peptide vaccine and to estimate progression-free survival (PFS) from vaccination and histologic diagnosis in patients newly diagnosed with GBM who expressed EGFRvIII.

\section{PATIENTS AND METHODS}

\section{Vaccine Product}

PEPvIII (LEEKKGNYVVTDHC) is a 13-amino-acid peptide with an additional terminal cysteine that spans the EGFRvIII mutation (AnaSpec, San Jose, CA). The peptide preparation was $>95 \%$ pure as assessed by highpressure liquid chromatography and was conjugated to keyhole limpet hemocyanin (KLH; biosyn, Carlsbad, CA) at a 1:1 ratio (w/w) (PEPvIII-KLH) using the heterobifunctional cross-linker sulfosuccinimidyl 6-[3'(2-pyridyldithio)propionamido]hexanoate (Pierce, Rockford, IL).

\section{Patient Selection}

Adults with newly diagnosed EGFRvIII-expressing GBM with a gross total resection $(>95 \%)$ and a Karnofsky performance status (KPS) $\geq 80 \%$ who had no radiographic evidence of progression after standard of care external beam radiation therapy and concurrent temozolomide (TMZ) and were willing to sign an informed consent were eligible for vaccination. The trial was approved by the US Food and Drug Administration (BB-IND-9944) and the local institutional review boards at Duke University Medical Center and M. D. Anderson Cancer Center.

\section{Clinical Protocol}

The initial three vaccinations of PEPvIII-KLH were given every two weeks starting 4 weeks after the completion of radiation. Subsequent vaccines were given once a month until radiographic evidence of tumor progression or death. All vaccines were given intradermally in the inguinal region within 10 $\mathrm{cm}$ of the inguinal ligament on alternating sides.

Patients were monitored once a month by physical examination and every two months by magnetic resonance imaging. Progressive disease was defined radiographically according to the MacDonald criteria ${ }^{19}$ or by the development of a new contrast-enhancing lesion of $>1 \mathrm{~cm}$ at the discretion of the treating neurooncologist. On tumor progression, further treatment was at the discretion of the patient's treating neurooncologist.

\section{Immunologic Monitoring and Immunohistochemical Analysis}

Delayed-type hypersensitivity (DTH) testing for cellular immune responses to PEPvIII $(1 \mathrm{mg} / \mathrm{mL})$, as well as to the recall antigens tetanus toxoid (NDC 49281-800-83 undiluted; Aventis Pasteur, Swiftwater, PA), Candida (\#M15 1:1000; Greer Laboratories, Lenoir, NC), and Trichophyton (\#M26, 1:1000; Greer), was performed by using standard intradermal injections in a volume of $100 \mu \mathrm{L}$. A positive skin test for all antigens was defined as $>5 \mathrm{~mm}$ induration within 48 to 72 hours.

Serum for assessing humoral responses was stored at $-20^{\circ} \mathrm{C}$ before analysis in an enzyme-linked immunosorbent assay or a PEPvIII-Dynabead (Invitrogen, Carlsbad, CA) assay, as described previously. ${ }^{20}$

Immunohistochemistry (IHC) for EGFR $v I I I$ was performed on paraffinembedded tissue, as previously described. ${ }^{7,21}$ IHC was performed in a Clinical Laboratory Improvement Amendments-certified laboratory using compliant techniques.

\section{Matched Cohort}

Patients in the matched cohort selected for PFS and OS comparisons were all treated contemporaneously at the University of Texas M. D. Anderson Cancer Center. In the matched control cohort $(n=17)$, all patients were adults; had EGFR $v I I I$-expressing primary GBMs, a KPS $\geq 80 \%$, and a resection of $>95 \%$ of the original tumor volume; and had been treated with radiation and TMZ. Patients with tumor progression within 4 weeks of completing radiation therapy were also excluded from this matched control cohort.

\section{Methylguanine Methyltransferase Promoter Methylation Status}

Samples were isolated from five sections of a representative tissue block. Following deparaffinization and proteinase K treatment, DNA was isolated from tumor tissue using a kit from Epicenter (Madison, WI). After sodium bisulfite treatment, methyl-specific polymerase chain reaction was performed as previously described. ${ }^{22}$ A ratio of methylated-to-unmethylated peaks of $\geq 1.0$ was scored as methylated.

\section{Statistical Analysis}

A two-stage clinical trial design was used to differentiate between a 6-month PFS rate of $20 \%$ and $40 \%$ from the time of vaccination with $\alpha$ and $\beta=.1$ on the basis of data derived from the matched cohort. If five or more of the 22 patients accrued in stage 1 were alive and free from disease progression at 6 months after vaccination, then an additional 22 patients were to be accrued during a second stage. If 11 or more of the 44 patients survived 6 months progression free from the time of vaccination, the treatment regimen was to be considered worthy of additional investigation. PFS and OS were estimated using Kaplan-Meier methods. The Cox proportional hazards model was used to compare PFS and OS of the vaccinated patients with the matched cohort; adjustments for known prognostic factors and covariates were made in the model. The study was not initially powered for these comparisons, however. Adverse events were defined according to the National Cancer Institute's Common Toxicity Criteria (Version 2.0). Frequencies were used to describe the EGFRvIII-specific antibody responses and DTH reactions. A binomial test was used to assess whether the proportion of patients with EGFRvIII IHC staining changes due to vaccination was significantly different from zero.

\begin{tabular}{|c|c|c|c|c|}
\hline \multirow[b]{2}{*}{ Parameter } & \multicolumn{2}{|c|}{$\begin{array}{c}\text { EGFRvIII } \\
\text { Vaccine Group }\end{array}$} & \multicolumn{2}{|c|}{$\begin{array}{c}\text { Matched } \\
\text { Cohort }\end{array}$} \\
\hline & No. & $\%$ & No. & $\%$ \\
\hline Total No. of Patients & 18 & & 17 & \\
\hline \multicolumn{5}{|l|}{ Age, years } \\
\hline Median & \multicolumn{2}{|c|}{52} & \multicolumn{2}{|c|}{59} \\
\hline Range & \multicolumn{2}{|c|}{$29-67$} & \multicolumn{2}{|c|}{$37-71^{*}$} \\
\hline Sex (male) & 13 & 72 & 8 & 47.1 \\
\hline \multicolumn{5}{|l|}{ KPS } \\
\hline 100 & 7 & 39 & 1 & 6 \\
\hline 90 & 7 & 39 & 9 & 53 \\
\hline 80 & 4 & 22 & 7 & $41+$ \\
\hline EGFRvIII expression & 18 & 100 & 17 & 100 \\
\hline \multicolumn{5}{|c|}{ Extent of surgical resection, \% } \\
\hline Median & \multicolumn{2}{|c|}{100} & \multicolumn{2}{|c|}{100} \\
\hline Range & \multicolumn{2}{|c|}{$95-100$} & \multicolumn{2}{|c|}{$95-100$} \\
\hline Radiation & 18 & 100 & 17 & 100 \\
\hline TMZ treatment & 17 & 94 & 17 & 100 \\
\hline \multicolumn{5}{|c|}{$\begin{array}{l}\text { Abbreviations: EGFRvIII, epidermal growth factor receptor variant III; KPS } \\
\text { Karnofsky performance status; TMZ, temozolomide. } \\
{ }^{*} P=.055 . \\
\dagger P=.016 .\end{array}$} \\
\hline
\end{tabular}




\begin{tabular}{|c|c|c|c|c|c|c|c|c|c|c|}
\hline \multirow[b]{2}{*}{ Patient } & \multirow[b]{2}{*}{ Age (years) } & \multirow[b]{2}{*}{ Sex } & \multirow[b]{2}{*}{ KPS } & \multirow[b]{2}{*}{$\begin{array}{c}\text { MGMT } \\
\text { Methylation }\end{array}$} & \multicolumn{2}{|c|}{ Humoral Response } & \multicolumn{2}{|c|}{ DTH Response } & \multirow[b]{2}{*}{$\begin{array}{c}\text { PFS } \\
\text { (months) }\end{array}$} & \multirow[b]{2}{*}{$\begin{array}{c}\text { OS } \\
\text { (months) }\end{array}$} \\
\hline & & & & & $\begin{array}{c}\text { Before } \\
\text { Vaccination }\end{array}$ & $\begin{array}{c}\text { After } \\
\text { Vaccination }\end{array}$ & $\begin{array}{c}\text { Before } \\
\text { Vaccination }\end{array}$ & $\begin{array}{c}\text { After } \\
\text { Vaccination }\end{array}$ & & \\
\hline 1 & 62 & $M$ & 90 & $\mathrm{ME}$ & N/A & N/A & - & - & 6.7 & 16.2 \\
\hline 2 & 49 & M & 80 & N/A & N/A & N/A & - & - & 6.4 & 18.0 \\
\hline 3 & 51 & M & 100 & N/A & N/A & N/A & - & - & 9.9 & 21.0 \\
\hline 4 & 48 & M & 100 & U & - & $+^{*}$ & - & + & $58.5+$ & $58.5 \neq$ \\
\hline 5 & 52 & M & 100 & ME & - & - & - & - & 13.5 & 22.6 \\
\hline 6 & 42 & M & 100 & $U$ & - & - & - & + & 14.9 & 35.8 \\
\hline 7 & 51 & M & 90 & $U$ & - & - & - & + & $54.6+$ & $54.6 \neq$ \\
\hline 8 & 33 & M & 100 & N/A & - & - & - & - & $53.7 \dagger$ & $53.7 \ddagger$ \\
\hline 9 & 46 & $\mathrm{~F}$ & 90 & ME & - & - & - & - & 15.4 & 34.9 \\
\hline 10 & 29 & M & 90 & N/A & - & - & - & N/A & 6.4 & 11.2 \\
\hline 11 & 64 & $M$ & 90 & U & N/A & $\mathrm{N} / \mathrm{A}$ & - & - & 12.0 & 21.6 \\
\hline 12 & 64 & M & 80 & $U$ & - & + & - & - & 27.6 & 44.1 \\
\hline 13 & 54 & $\mathrm{~F}$ & 90 & N/A & - & - & - & - & 17.6 & 26.0 \\
\hline 14 & 63 & M & 80 & $\mathrm{ME}$ & - & + & - & - & 10.9 & 20.8 \\
\hline 15 & 67 & $\mathrm{~F}$ & 80 & $\mathrm{ME}$ & - & + & - & - & 6.5 & 13.4 \\
\hline 16 & 53 & $\mathrm{~F}$ & 100 & $\mathrm{ME}$ & - & + & - & - & 30.0 & 47.7 \\
\hline 17 & 52 & M & 100 & $\mathrm{ME}$ & - & + & - & - & 5.4 & $47.4 \ddagger$ \\
\hline 18 & 64 & $\mathrm{~F}$ & 90 & U & - & - & - & - & 16.4 & 23.1 \\
\hline \multicolumn{11}{|c|}{$\begin{array}{l}\text { Abbreviations: KPS, Karnofsky performance status; MGMT, methylguanine methyltransferase; DTH, delayed-type hypersensitivity; PFS, progression-free survival; } \\
\text { OS, overall survival; M, male; ME, methylated; N/A, sample not available; U, unmethylated; F, female; }(-) \text {, no response; }(+) \text {, positive response. } \\
\text { "Positive humoral response in CSF. } \\
\text { †No progression. } \\
\text { †Alive. }\end{array}$} \\
\hline
\end{tabular}

\section{RESULTS}

\section{Study Population}

At both centers, all known patients with EGFRvIII-expressing newly diagnosed GBM were screened for study eligibility. Only two patients at each site refused participation despite being eligible. Twenty-one patients were enrolled and vaccinated before accrual was suspended at the end of stage 1 . On retrospective quality control review, three patients were found not to meet eligibility criteria because $<95 \%$ of the tumor volume had been resected. Therefore, the primary focus of analyses reported here will involve the 18 eligible patients. Follow-up through October 2008 is reflected in this article. All but one of the patients who were vaccinated received TMZ delivered concurrently with radiotherapy ${ }^{2}$ (Table 1). TMZ therapy after radiation was not used in conjunction with the vaccinations. Study data are summarized in Table 2.

\section{Toxicity and Adverse Events}

Toxicity was generally minimal (Appendix Table A1, online only) and mostly related to injection site reactions, which never produced grade $\geq 2$ toxicity. However, one patient was removed from the study when a presumed severe allergic reaction (numbness and tingling in the perioral area) to the vaccine components was suspected, although a similar event occurred several weeks later when the patient had an unrelated procedure. This was still considered a serious adverse event. In addition, one patient developed asymptomatic areas of T2signal hyperintensity that on subsequent magnetic resonance imaging scans demonstrated contrast enhancement that eventually resolved. These lesions remained hypometabolic on positron emission tomography. This reaction was deemed a grade 1 leukoencephalopathy toxicity (Appendix Table A1 and Fig 1) and resolved without treatment.

\section{PFS}

The median PFS from time of histologic diagnosis for the 18 eligible patients was 14.2 months (95\% CI, 9.9 to 17.6 months; Fig 2A and Appendix Table A2, online only). Among the 18 eligible patients, 12 patients $(67 \% ; 95 \% \mathrm{CI}, 40 \%$ to $83 \%)$ were alive and without radiographic evidence of progression 6 months after vaccination, and 17 patients (94\%; 95\% CI, 67\% to $99 \%$ ) were alive and without radiographic evidence of progression 6 months after histologic diagnosis (Appendix Table A2). After considering these data, we rejected the null hypothesis for the primary end point. Accrual was terminated after this interim analysis because the number of patients
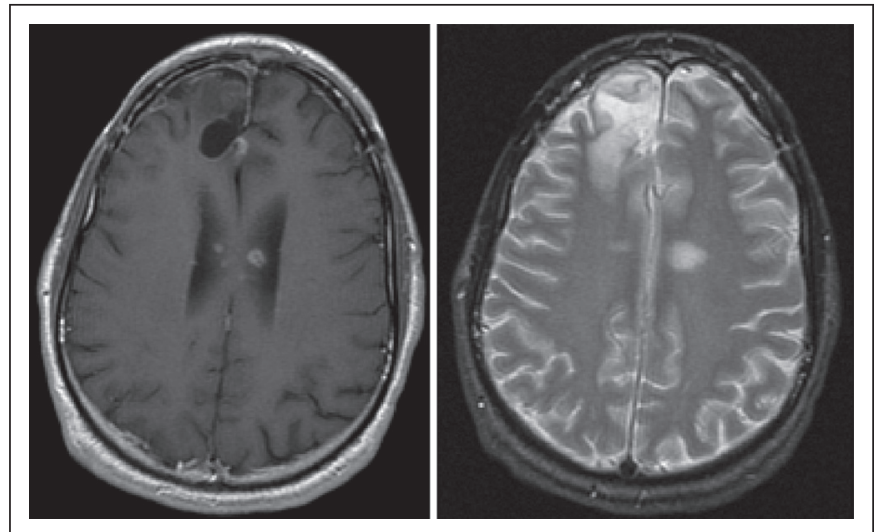

Fig 1. Axial magnetic resonance images showing progression of multiple lesions surrounding the corpus callosum. Left: T1-weighted, contrast-enhanced image 8 months from first vaccination showing new enhancing lesions distant from the right frontal tumor cavity. Right: T2-weighted image showing hyperintense lesion in similar distribution. Areas of contrast-enhancement have resolved, but areas of T2 hyperintensity have persisted. 


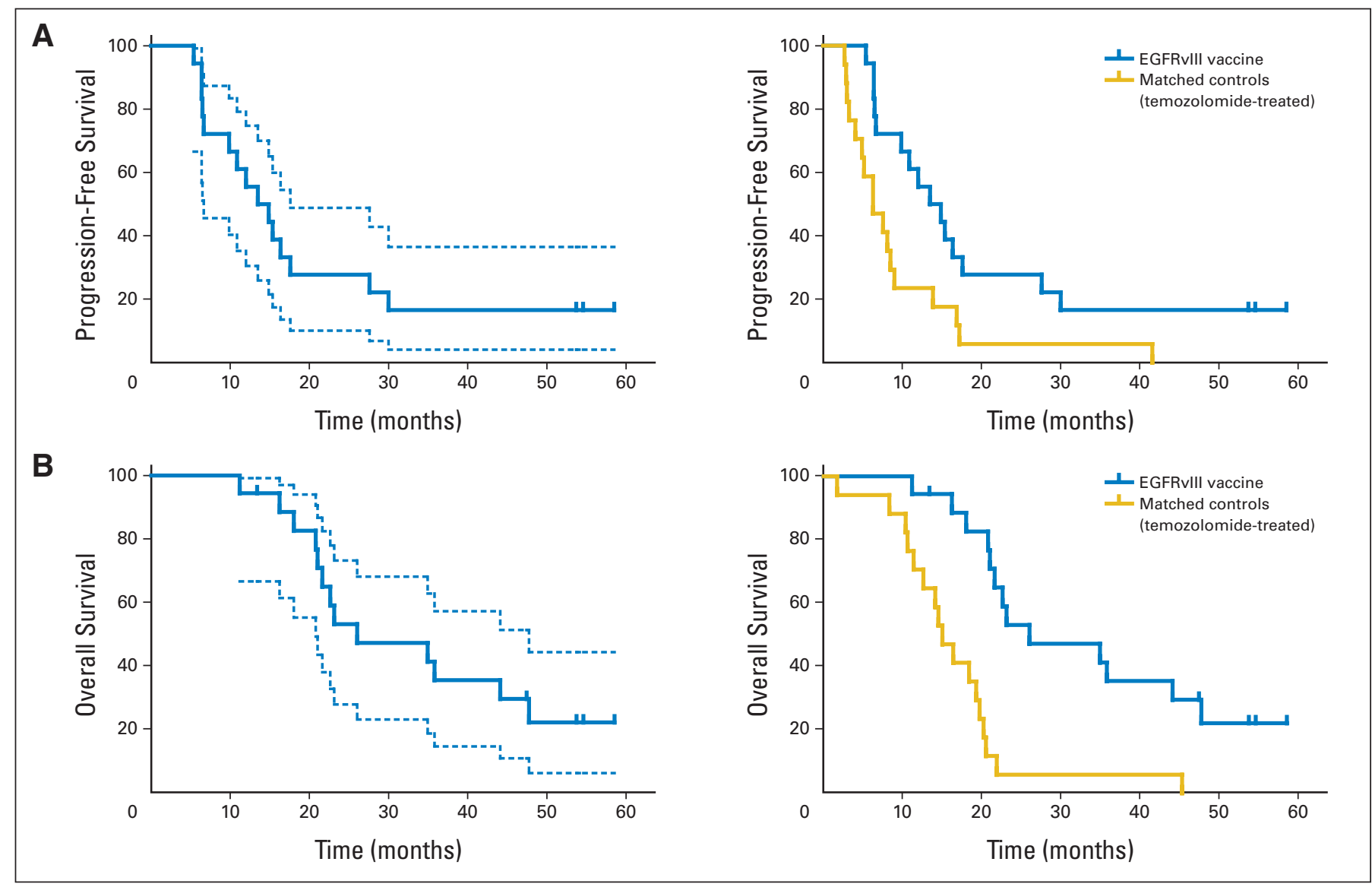

Fig 2. (A) Progression-free survival (PFS). Left: The median PFS from histologic diagnosis in the patients who had been vaccinated ( $n=18$; solid blue line) was 14.2 months (95\% Cl, 9.9 to 17.6 months). Dotted blue lines show 95\% Cls. Right: In the temozolomide (TMZ) -treated historical cohort ( $n=17$; gold line), the median PFS was 6.3 months $(95 \% \mathrm{Cl}, 4.1$ to 9.0 months). The PFS of patients who had been vaccinated compares favorably with that of the TMZ-treated cohort before ( $P=.013)$ and after ( $P=.041)$ adjustment for age and Karnofsky performance status (KPS). (B) Overall survival (OS). Left: The median survival of patients who had been vaccinated ( $n=18$; solid blue line) was 26.0 months ( $95 \%$ Cl, 21.0 to 47.7 months;). Dotted blue lines show 95\% Cls. Right: In the TMZ-treated historical cohort ( $\mathrm{n}=17$; gold line), the median survival was 15.0 months ( $95 \% \mathrm{Cl}, 11.4$ to 19.7 months). The OS of patients who had been vaccinated compares favorably with that of the TMZ-treated cohort before $(P=.002)$ and after $(P=.001)$ adjustment for age and KPS. EGFRVIII, epidermal growth factor receptor variant III.

living progression free for more than 6 months after vaccination $(\mathrm{n}=$ 12) exceeded the critical value associated with the hypothesis test to be conducted at the end of the second stage of the study $(\mathrm{n} \geq 11)$.

In the matched cohort $(n=17)$, the median PFS from histologic diagnosis was 6.3 months (95\% CI, 4.1 to 9.0 months). The PFS hazard ratio (HR) comparing the matched cohort with our patients was 2.4 (95\% CI, 1.2 to $5.0 ; P=.013$; Fig 2A). Even after adjustment for age and KPS, the PFS of vaccinated patients remained significantly greater than that observed in the TMZ-treated matched control group (HR, 2.2; $95 \% \mathrm{CI}, 1.0$ to $4.8 ; P=.041)$. Inferences were not affected by the exclusion of the three ineligible patients.

\section{Survival Time}

The median OS from time of histologic diagnosis for the 18 eligible patients was 26.0 months (95\% CI, 21.0 to 47.7 months). Among the 18 eligible patients, $94 \%$ (95\% CI, 67\% to 99\%) were alive 12 months after vaccination and $94 \%$ (95\% CI, 67\% to 99\%) were alive at 12 months after histologic diagnosis (Fig 2B and Appendix Table A2). In the matched cohort $(n=17)$, time from histologic diagnosis to the median OS was 15.0 months (95\% CI, 11.4 to 19.7 months). After adjustment for age and KPS, the survival of vaccinated patients was significantly better than that observed in a TMZ-treated matched control group (HR, 5.1; 95\% CI, 1.9 to $13.9 ; P=.001$ ). Inferences were not affected by the exclusion of the three ineligible patients.

\section{IHC Analysis of EGFRvIII Expression}

Among recurrent tumors from which pathologic material was obtained $(\mathrm{n}=11)$, all were evaluated by IHC for EGFRvIII expression. Of these 11 samples, $82 \%$ (95\% CI, 48\% to 97\%) had lost EGFR $v I I I$ expression at recurrence (binomial test $P<.001$; Table 3 ; Fig 3 ). Of the two patients who had positive EGFR $v$ III staining at recurrence, one had $<1 \%$ of cells staining for EGFR $v I I$.

\section{Immune Responses}

At the interim analysis when the study was terminated, $14 \mathrm{pa}-$ tients had serum samples that had been analyzed for EGFR $v I I I$-specific humoral responses. Six (43\%; 95\% CI, 18\% to $71 \%$; $P<.001$ ) had evidence of a humoral response against PEPvIII. The maximum concentration of antibody that reacted against EGFR $v I I I$, as estimated by comparison with an EGFRvIII-specific monoclonal antibody, ${ }^{20}$ was 
Table 3. EGFRvII/ Immunohistochemistry Before and After Vaccination

\begin{tabular}{cl}
\hline Before Vaccination & At Recurrence \\
\hline Positive & Negative \\
Positive & Negative \\
Positive & Negative \\
Positive & Positive $(<1 \%)$ \\
Positive & Negative \\
Positive & Negative \\
Positive & Negative \\
Positive & Negative \\
Positive & Negative \\
Positive & Positive \\
Positive & Negative
\end{tabular}

NOTE. Percent negative after vaccine is $82 \%(95 \% \mathrm{Cl}, 48 \%$ to $97 \%)$ or nine of 11 ; binomial test $P<.001$.

Abbreviation: EGFRvIII, epidermal growth factor receptor variant III.

$910 \mathrm{ng} / \mathrm{mL}$. In one patient with a positive serum titer, CSF was obtained and it demonstrated a concentration of antibody against EGFR$v I I I$ of $16.2 \mathrm{ng} / \mathrm{mL}$.

The median OS from histologic diagnosis for the six patients who developed EGFR $v I I$-specific antibody responses was 47.7 months (95\% CI, 20.8 to $\infty$ months; Fig 4 ). For the eight patients who did not develop antibody responses, the OS was only 22.8 months (95\% CI, 21.0 to 34.9 months). After adjustment for age, KPS, and methylguanine methyltransferase (MGMT) methylation, the OS from vaccination $(P=.025)$ and histologic diagnosis $(P=$ .025 ) of the patients who developed antibody responses was found to be greater than the OS for those who had not. However, these findings need to be validated prospectively in a model with a larger number of patients.

To assess patient cellular immune responses to the vaccinating antigen and endogenous antigens in vivo, DTH skin tests were performed with PEPvIII and recall antigens. All patients showed no response to PEPvIII before vaccination. After vaccination, three $(18 \%$; $95 \%$ CI, $4 \%$ to $43 \%$; binomial proportions $P<.001)$ of 17 showed a positive DTH response to PEPvIII after vaccination.

The median OS from histologic diagnosis for the three patients who developed PEPvIII-specific DTH responses had not been reached at 50 months follow-up. For the 14 patients who did not develop DTH responses to PEPvIII, the OS was 23.1 months (95\% CI, 21.0 to 44.1 months). Although the number of patients who developed PEPvIII DTH responses was small, the patients did have a significantly longer PFS and OS from vaccination and histologic diagnosis $(P=.03)$. Conversely, DTH responses to recall antigens did not have a significant effect on PFS from vaccination $(P=.81)$ or histologic diagnosis $(P=.88)$ or on OS from vaccination $(P=.58)$ or histologic diagnosis $(P=.61)$. These small patient numbers, however, preclude meaningful adjustment for known prognostic factors such as age, KPS, and MGMT methylation.

\section{MGMT Methylation}

Because almost all of our study patients were treated with concurrent TMZ during radiotherapy, an unintentional study bias may have been introduced by selecting patients with GBM that possessed the capacity for MGMT methylation, which compromises DNA repair and is associated with longer survival in patients with GBM who receive alkylating agents such as TMZ. ${ }^{22}$ Of the patients from whom tumor tissue was obtained for MGMT testing $(\mathrm{n}=13)$, seven $(54 \%)$ had MGMT methylation, which is a slightly higher proportion than
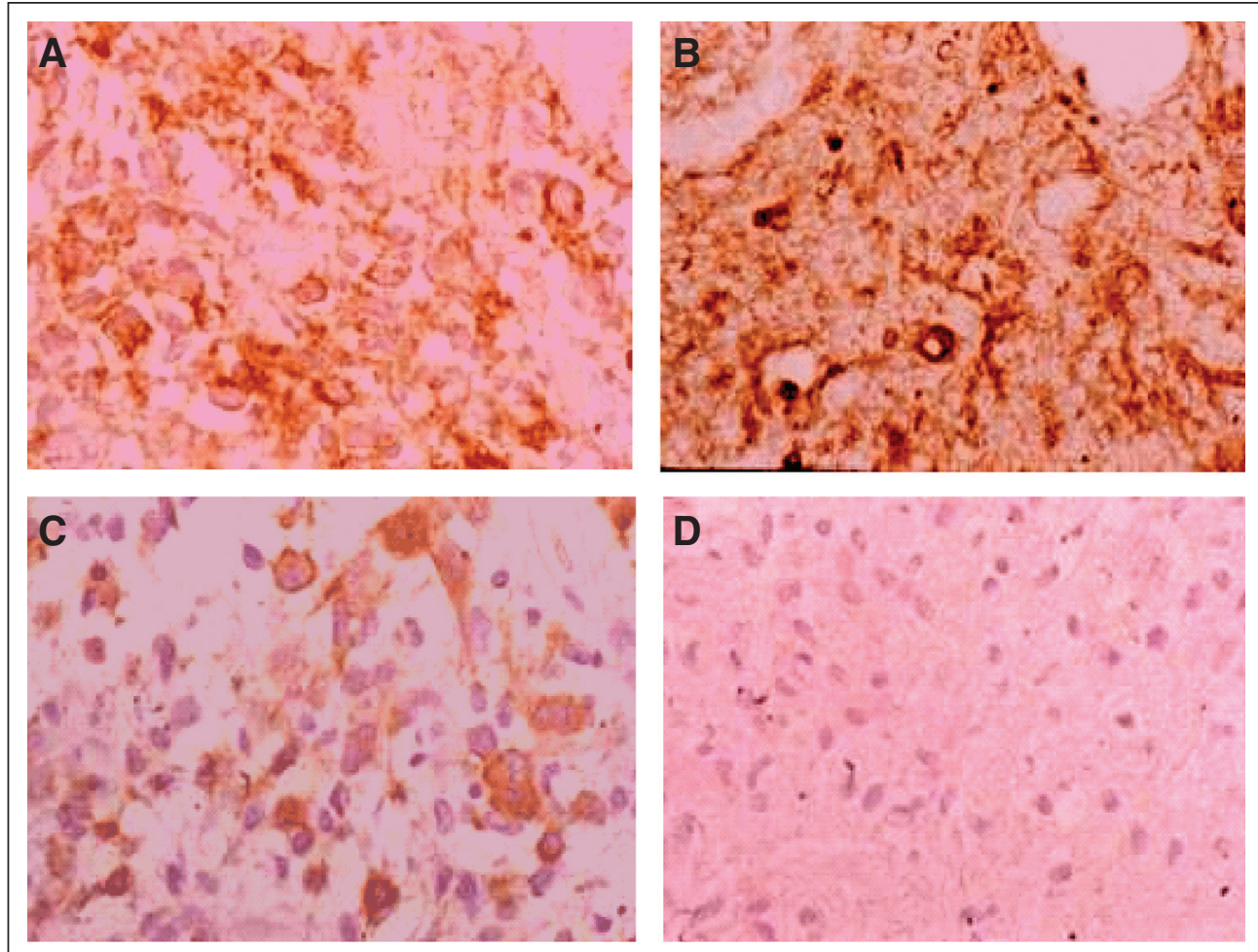

Fig 3. Epidermal growth factor receptor (EGFR) and EGFR variant III (EGFRvIII) immunohistochemistry of a patient with glioblastoma multiforme (GBM). Staining with (A) EGFR and (B) EGFRvIll before vaccine. (C) Preservation of EGFR staining but (D) specific loss of EGFRvII/ staining at recurrence after vaccination. 

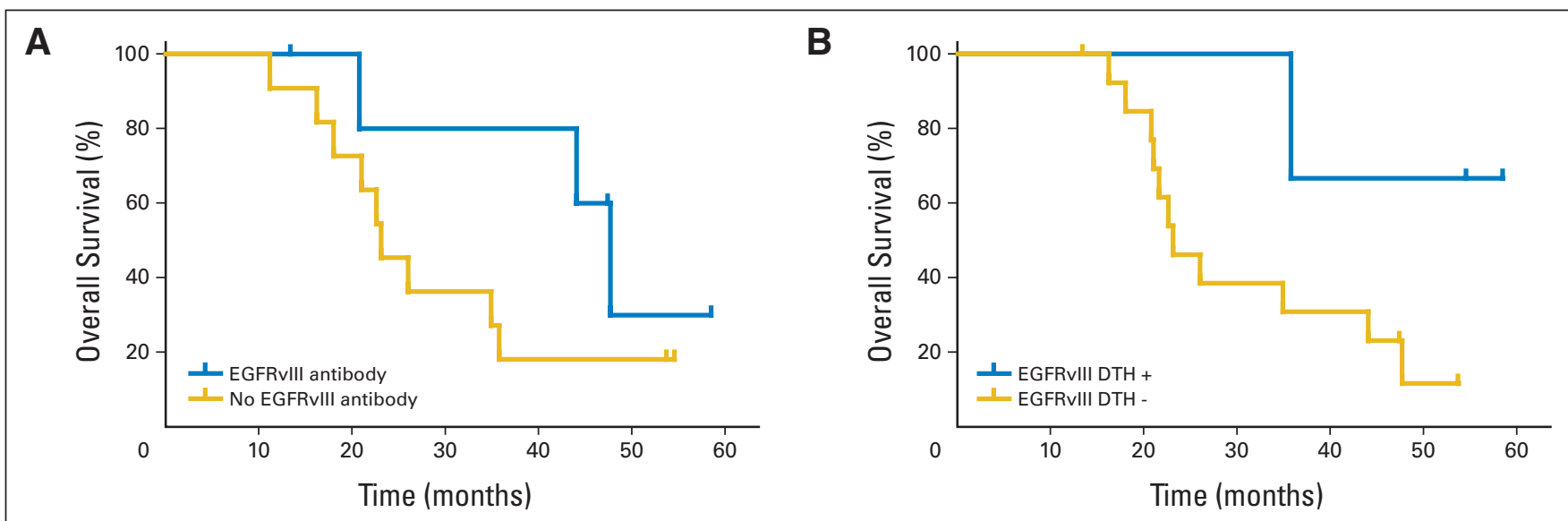

Fig 4. Immune response correlates. Overall survival (OS) from histologic diagnosis for all patients for whom serum was available to test for epidermal growth factor

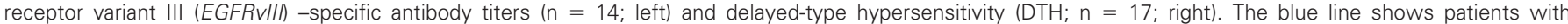

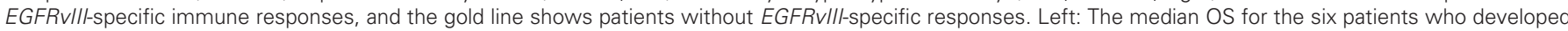

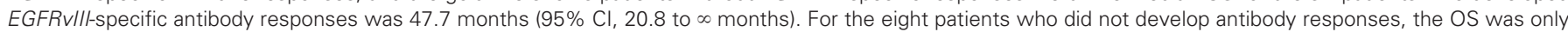

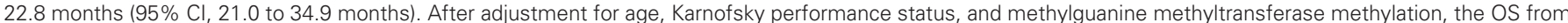

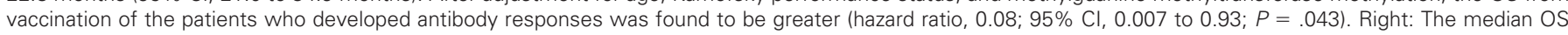

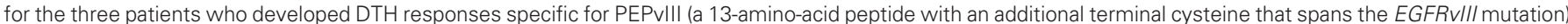

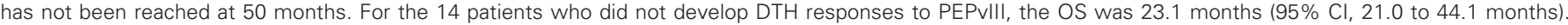
Patients who developed PEPVIII DTH responses had a significantly longer OS $(P=.03)$.

previously reported. ${ }^{22}$ However, the PFS and OS from either histologic diagnosis or vaccination is actually unexpectedly longer in our study for patients with unmethylated MGMT. This does not reach statistical significance in unadjusted univariate analyses but when adjusted for age and KPS, patients with unmethylated MGMT had a significantly longer PFS from vaccination (HR, 0.17; 95\% CI, 0.032 to $0.90 ; P=.037$ ) and histologic diagnosis (HR, 0.17; 95\% CI, 0.032 to $0.90 ; P=.037)$ than patients with methylated MGMT who were treated with TMZ. There was also a trend for these patients to have a longer OS from vaccination $(P=.062)$ and histologic diagnosis $(P=.062)$. These data are summarized in Appendix Figure A1 and Appendix Table A3 (online only). A larger number of patients is needed, however, to validate these observations.

\section{DISCUSSION}

Our study results demonstrate that vaccinating patients who have newly diagnosed EGFR $v I I I$-positive GBM with a peptide containing an EGFR $v I I I$-specific epitope is safe, induces specific immunity against EGFRvIII, and is associated with the elimination of EGFR $v I I$-expressing cells at recurrence. This observation that an EGFRvIII-targeted vaccine is capable of potentially eliminating EGFR $v I I I$-expressing tumor cells in the majority of patients is an intriguing finding and consistent with what we found in our preclinical studies in mice. ${ }^{18}$ It suggests that the immunologic privilege of the brain may not be absolute in this context as well. It is difficult to differentiate between elimination of a specific population of tumor cells expressing EGFR $v I I$ and a downregulation of the expression of the mutated tyrosine kinase, however. Recurrent tumors in these patients continue to express the wild-type EGFR protein. This raises the possibility that intramolecular cross-priming that might induce immune responses against wild-type EGFR may be attenuated.
We also found that patients with GBM who had received the PEPvIII-KLH vaccine had significantly longer OS than a contemporaneously treated cohort matched for eligibility criteria and treatment, and they compare favorably with recently published trials evaluating other patient cohorts treated with carmustine polymers (13.7 months $)^{23}$ or serial TMZ cycles (14.6 months). ${ }^{2}$ Although the median OS time observed in this study is encouraging, it may not be different from an untreated population or from that reported in other recent studies using different immunotherapy approaches. ${ }^{24-28}$ Although nearly consecutive patients with EGFR $I I I$-expressing GBM were enrolled at two different centers during this trial, the small sample size and restricted eligibility criteria, although chosen to optimize the identification of an immune response, led to some bias that cannot be adequately addressed despite our attempts to select an adequate matched control population. Thus, although these data suggest that vaccination with PEPvIII-KLH may improve PFS and OS in this population, definitive evidence of efficacy will require a blinded, placebo-controlled, randomized phase III study, which is currently in the planning stages.

Our finding that vaccinated patients with an unmethylated MGMT promoter, which usually confers resistance to TMZ, ${ }^{22}$ actually had a longer PFS and OS than those patients with methylated MGMT raises the possibility that EGFR $I I I$-targeted vaccination may be an effective alternative for those patients and mandates that phase III trials of this vaccine approach will need to stratify patients for this variable. Although unexpected, this finding is consistent with the observation by Murat et $\mathrm{al}^{29}$ that EGFR signaling, which would be induced in EGFR $v I I I$-expressing tumor cells, confers resistance to TMZ and predicts a poor outcome after standard therapy.

Although this study demonstrates the possible benefits of vaccination with a peptide that contains a tumor-specific epitope, there remain a number of issues that must be addressed to optimize this therapeutic modality. While one distinct advantage of our approach is 
that the vaccine is available off-the-shelf and does not involve laborintensive and expensive cell preparation techniques, a notable disadvantage is that only a subset of patients with GBM expresses the targeted antigen, EGFRvIII. Thus, although a tumor-specific vaccine may have the advantage of minimizing autoimmune complications, the heterogeneity of malignant brain tumors may limit the effectiveness of vaccinations that target only one tumor-specific antigen. Vaccines that target only one antigen may not target all tumors or all cells comprising a tumor and may therefore select for the survival and proliferation of those cells that do not express the targeted antigen. This may ultimately limit this potentially promising approach. Multiantigenic vaccines may serve as an alternative, but they risk the induction of autoimmunity. Using such an approach, however, several investigators have demonstrated robust immunologic responses and encouraging clinical results without catastrophic autoimmune responses. ${ }^{26-28,30,31}$

\section{AUTHORS' DISCLOSURES OF POTENTIAL CONFLICTS OF INTEREST}

Although all authors completed the disclosure declaration, the following author(s) indicated a financial or other interest that is relevant to the subject matter under consideration in this article. Certain relationships marked with a " $U$ " are those for which no compensation was received; those relationships marked with a " $C$ " were compensated. For a detailed description of the disclosure categories, or for more information about ASCO's conflict of interest policy, please refer to the Author Disclosure Declaration and the Disclosures of Potential Conflicts of Interest section in Information for Contributors.

Employment or Leadership Position: None Consultant or Advisory Role: John H. Sampson, Celldex Therapeutics (C); Amy B. Heimberger, Celldex Therapeutics (C); Mark R. Gilbert, Genentech (C), Merck (C), Pfizer (C); Darell D. Bigner, Celldex Therapeutics (C) Stock Ownership: None Honoraria: John H. Sampson, Celldex Therapeutics; Mark R. Gilbert, Merck, Genentech Research Funding: John H. Sampson,
Celldex Therapeutics; Mark R. Gilbert, Schering-Plough, Genentech Expert Testimony: None Other Remuneration: John H. Sampson and Darell D. Bigner received funding under the Duke University Faculty Plan from license fees paid to Duke University by Celldex Therapeutics.

\section{AUTHOR CONTRIBUTIONS}

Conception and design: John H. Sampson, Amy B. Heimberger, Gary E. Archer, Kenneth D. Aldape, Allan H. Friedman, Henry S. Friedman, Mark R. Gilbert, Roger E. McLendon, Duane A. Mitchell, David A. Reardon, Raymond Sawaya, Weiming Shi, James J. Vredenburgh, Darell D. Bigner

Financial support: John H. Sampson, Amy B. Heimberger

Administrative support: John H. Sampson, Amy B. Heimberger Provision of study materials or patients: John H. Sampson, Amy B. Heimberger, Gary E. Archer, Kenneth D. Aldape, Allan H. Friedman, Henry S. Friedman, Mark R. Gilbert, Roger E. McLendon, David A. Reardon, Raymond Sawaya, Weiming Shi, James J. Vredenburgh, Darell D. Bigner

Collection and assembly of data: John H. Sampson, Amy B.

Heimberger, Gary E. Archer, Kenneth D. Aldape, Mark R. Gilbert, Roger E. McLendon, Duane A. Mitchell, Raymond Sawaya, Robert J. Schmittling, Weiming Shi, James J. Vredenburgh, Darell D. Bigner Data analysis and interpretation: John H. Sampson, Amy B. Heimberger, Gary E. Archer, Kenneth D. Aldape, Mark R. Gilbert, James E. Herndon II, Roger E. McLendon, Duane A. Mitchell, Raymond Sawaya, Robert J. Schmittling, Weiming Shi, Darell D. Bigner Manuscript writing: John H. Sampson, Amy B. Heimberger, Gary E. Archer, Kenneth D. Aldape, Allan H. Friedman, Henry S. Friedman, Mark R. Gilbert, James E. Herndon II, Roger E. McLendon, Duane A. Mitchell, David A. Reardon, Raymond Sawaya, Robert J. Schmittling, Weiming Shi, James J. Vredenburgh, Darell D. Bigner

Final approval of manuscript: John H. Sampson, Amy B. Heimberger, Gary E. Archer, Kenneth D. Aldape, Allan H. Friedman, Henry S. Friedman, Mark R. Gilbert, James E. Herndon II, Roger E. McLendon, Duane A. Mitchell, David A. Reardon, Raymond Sawaya, Robert J. Schmittling, Weiming Shi, James J. Vredenburgh, Darell D. Bigner

\section{REFERENCES}

1. Imperato JP, Paleologos NA, Vick NA: Effects of treatment on long-term survivors with malignant astrocytomas. Ann Neurol 28:818-822, 1990

2. Stupp R, Mason WP, van den Bent MJ, et al: Radiotherapy plus concomitant and adjuvant temozolomide for glioblastoma. N Engl J Med 352:987996, 2005

3. Wong AJ, Ruppert JM, Bigner $\mathrm{SH}$, et al: Structural alterations of the epidermal growth factor receptor gene in human gliomas. Proc Natl Acad Sci U S A 89:2965-2969, 1992

4. Heimberger AB, Hlatky R, Suki D, et al: Prog nostic effect of epidermal growth factor receptor and EGFRvIII in glioblastoma multiforme patients. Clin Cancer Res 11:1462-1466, 2005

5. Ge H, Gong X, Tang CK: Evidence of high incidence of EGFRvIII expression and coexpression with EGFR in human invasive breast cancer by laser capture microdissection and immunohistochemical analysis. Int J Cancer 98:357-361, 2002

6. Sok JC, Coppelli FM, Thomas SM, et al: Mutant epidermal growth factor receptor (EGFRvIII) contributes to head and neck cancer growth and resistance to EGFR targeting. Clin Cancer Res 12: 5064-5073, 2006
7. Wikstrand CJ, Hale LP, Batra SK, et al: Monoclonal antibodies against EGFRvIII are tumor specific and react with breast and lung carcinomas and malignant gliomas. Cancer Res 55:3140-3148, 1995

8. Purev $E$, Cai $D$, Miller $E$, et al: Immune responses of breast cancer patients to mutated epidermal growth factor receptor (EGF-RvIII, Delta EGF-R, and de2-7 EGF-R). J Immunol 173:64726480, 2004

9. Humphrey PA, Wong AJ, Vogelstein B, et al: Anti-synthetic peptide antibody reacting at the fusion junction of deletion-mutant epidermal growth factor receptors in human glioblastoma. Proc Nat Acad Sci U S A 87:4207-4211, 1990

10. Chu CT, Everiss KD, Wikstrand $C J$, et al: Receptor dimerization is not a factor in the signalling activity of a transforming variant epidermal growth factor receptor (EGFRVIII). Biochem J 324:855-861, 1997

11. Batra SK, Castelino-Prabhu S, Wikstrand CJ, et al: Epidermal growth factor ligand-independent, unregulated, cell-transforming potential of a naturally occurring human mutant EGFRvIll gene. Cell Growth Differ 6:1251-1259, 1995

12. Lal A, Glazer CA, Martinson HM, et al: Mutant epidermal growth factor receptor up-regulates molecular effectors of tumor invasion. Cancer Res 62:3335-3339, 2002
13. Boockvar JA, Kapitonov D, Kapoor G, et al: Constitutive EGFR signaling confers a motile phenotype to neural stem cells. Mol Cell Neurosci 24: 1116-1130, 2003

14. Nagane M, Coufal F, Lin $\mathrm{H}$, et al: A common mutant epidermal growth factor receptor confers enhanced tumorigenicity on human glioblastoma cells by increasing proliferation and reducing apoptosis. Cancer Res 56:5079-5086, 1996

15. Lammering G, Valerie $K$, Lin PS, et al: Radiation-induced activation of a common variant of EGFR confers enhanced radioresistance. Radiother Oncol 72:267-273, 2004

16. Montgomery RB, Guzman J, O'Rourke DM, et al: Expression of oncogenic epidermal growth factor receptor family kinases induces paclitaxel resistance and alters beta-tubulin isotype expression. J Biol Chem 275:17358-17363, 2000

17. Heimberger AB, Archer GE, Crotty LE, et al: Dendritic cells pulsed with a tumor-specific peptide induce long-lasting immunity and are effective against murine intracerebral melanoma. Neurosurgery 50:158-166, 2002

18. Heimberger AB, Crotty LE, Archer GE, et al: Epidermal growth factor receptor VIII peptide vaccination is efficacious against established intracerebral tumors. Clin Cancer Res 9:4247-4254 2003 
19. Macdonald DR, Cascino TL, Schold SC Jr, et al: Response criteria for phase II studies of supratentorial malignant glioma. J Clin Oncol 8:1277-1280, 1990

20. Schmittling RJ, Archer GE, Mitchell DA, et al: Detection of humoral response in patients with glioblastoma receiving EGFRvIII-KLH vaccines. J Immunol Methods 339:74-81, 2008

21. Pelloski CE, Ballman KV, Furth $A F$, et al: Epidermal growth factor receptor variant III status defines clinically distinct subtypes of glioblastoma. J Clin Oncol 25:2288-2294, 2007

22. Hegi ME, Diserens AC, Gorlia T, et al: MGMT gene silencing and benefit from temozolomide in glioblastoma. N Engl J Med 352:997-1003, 2005

23. Westphal M, Hilt DC, Bortey E, et al: A phase 3 trial of local chemotherapy with biodegradable carmustine (BCNU) wafers (Gliadel wafers) in patients with primary malignant glioma. Neuro Oncol 5:79-88, 2003
24. Wheeler CJ, Black KL, Liu G, et al: Vaccination elicits correlated immune and clinical responses in glioblastoma multiforme patients. Cancer Res 68 : 5955-5964, 2008

25. De Vleeschouwer S, Fieuws S, Rutkowski S, et al: Postoperative adjuvant dendritic cell-based immunotherapy in patients with relapsed glioblastoma multiforme. Clin Cancer Res 14:3098-3104, 2008

26. Yu JS, Liu G, Ying $H$, et al: Vaccination with tumor lysate-pulsed dendritic cells elicits antigenspecific, cytotoxic T-cells in patients with malignant glioma. Cancer Res 64:4973-4979, 2004

27. Yu JS, Wheeler CJ, Zeltzer PM, et al: Vaccination of malignant glioma patients with peptide-pulsed dendritic cells elicits systemic cytotoxicity and intracranial T-cell infiltration. Cancer Res 61:842-847, 2001

28. Liau LM, Prins RM, Kiertscher SM, et al: Dendritic cell vaccination in glioblastoma patients induces systemic and intracranial T-cell responses modulated by the local central nervous system tumor microenvironment. Clin Cancer Res 11:55155525, 2005

29. Murat A, Migliavacca E, Gorlia T, et al: Stem cell-related "self-renewal" signature and high epidermal growth factor receptor expression associated with resistance to concomitant chemoradiotherapy in glioblastoma. J Clin Oncol 26:3015-3024, 2008

30. Kikuchi T, Akasaki $Y$, Abe $T$, et al: Vaccination of glioma patients with fusions of dendritic and glioma cells and recombinant human interleukin 12 . J Immunother 27:452-459, 2004

31. Rutkowski S, De Vleeschouwer S, Kaempgen E, et al: Surgery and adjuvant dendritic cell-based tumour vaccination for patients with relapsed malignant glioma, a feasibility study. Br J Cancer 91:16561662, 2004

\section{Join the conversation at ASCOconnection.org}

ASCOconnection.org is ASCO's newly launched social networking site. As the premier online meeting place for the worldwide oncology community, ASCOconnection.org gives you a platform to:

Share ideas and opinions with other ASCO members

Expand your network of contacts across the oncology community

Interact directly with ASCO leadership

ASCOconnection.org is your online home for exchanging oncology-related information, news, and experiences with colleagues around the world.

- Read and reply to a blog

- Start or join a discussion forum

- Share your knowledge and experiences

- Make new contacts and find new collaborators

ASCOconnection.org bloggers include: ASCO President George W. Sledge, MD; Immediate Past President Douglas W. Blayney, MD; Journal of Oncology Practice Editor-in-Chief John V. Cox, DO, FACP; and other ASCO leaders.

Log in today at ASCOconnection.org and get connected. 